\title{
MACROMASTIA: A RISK FACTOR FOR CARPAL TUNNEL SYNDROME?
}

\author{
Ana Silva Guerra, Carlos Marques Correia, \\ José Manuel Videira e Castro and Maria Angélica Almeida \\ Plastic Surgery Department, S. José Hospital, Lisbon, Portugal
}

Received 17 November 2010; Revised 9 March 2011; Accepted 9 March 2011

\begin{abstract}
Purpose: To evaluate the prevalence of carpal tunnel syndrome (CTS) in a cohort of women with macromastia and to assess the evolution of the CTS signs and symptoms after breast surgery.

Population: A series of 123 women was evaluated. CTS was defined by co-existence of symptoms, two physical findings and electrophysiological evaluation. One year after reduction mammaplasty, the 22 patients who had been diagnosed CTS were re-evaluated.

Results: CTS group of women had a mean age of 38.8 years, mean body mass index of $28.5 \mathrm{~kg} / \mathrm{m}^{2}$ and mean breast size of $35.9 \mathrm{~cm}$. Age and nipple-to-sternal notch distance were statistically significantly associated with CTS $(p=0.001$ and $p=0.001$, respectively). A year after surgery 15 patients were re-assessed: nine patients reported absence or improvement of CTS symptoms while six patients reported symptoms persistence or worsening.

Conclusion: Age, breast size, but not body mass index, have a positive correlation with the CTS.
\end{abstract}

Keywords: Macromastia; Carpal Tunnel Syndrome; Mammaplasty Reduction.

\section{INTRODUCTION}

Macromastia is a commonly reported disorder among women and appears to be a multifactorial phenomenon with a hereditary component and uncertain correlation with bodyweight. ${ }^{1-6}$ Symptoms of female breast hypertrophy are well recognized and the majority involves musculoskeletal discomfort (back pain, neck and shoulder pain) attributable to poor posture due to the excessive weight of the breasts. ${ }^{1-5}$ Poor hygiene in the submammary crease leads to irritations, rashes, and even cutaneous infections. Psychological issues and personal embarrassment are also described specially in younger patients. Older patients frequently refer concern relating to the increased risk for developing malignant breast tumor due to the higher breast volume. ${ }^{7}$ Occasionally upper limb paresthesia has also been mentioned and recent reports concentrate on the potential causal relation between breast size and neurological upper limb symptoms. ${ }^{3,4,8-10}$

Carpal tunnel syndrome (CTS), the most common of all entrapment syndromes, is an important cause of pain and functional impairment of the hand. It is known to be causally related to congenital, traumatic, metabolic/endocrine, inflammatory, infectious, or idiopathic causes. ${ }^{11}$ In addition, repetitive use is thought to increase the incidence of CTS. ${ }^{12}$ The prevalence of electrophysiological confirmed CTS among women is $3 \%$ who are affected three times as often as men. ${ }^{4,11,13}$ The association between macromastia and upper limb entrapment 
syndromes remain controversial. Previous studies have reported that $15 \%$ to $22.6 \%$ of women with macromastia have neurological complains in median and ulnar hand territories. ${ }^{8-10,14}$ Recently, two articles described an increased incidence (19.9\% and 22\%) of CTS syndrome in a group of women with macromastia. ${ }^{3,4}$

The purpose of this study was to assess the prevalence of CTS in a cohort of women with macromastia requesting bilateral breast reduction, over a three-year period, at a plastic surgery department. It also sought to assess the evolution of the CTS signs and symptoms after breast surgery.

\section{MATERIAL AND METHODS}

Between January 2005 and December 2008, a consecutive series of 123 women with macromastia requesting bilateral breast reduction were evaluated. Exclusion criteria were: previous upper limb surgery, known osteoarthritis of the cervical spine and upper limb, diabetes, autoimmune disease and thyroid disease. Twenty-six patients were excluded: two had previous upper limb trauma surgery, five had osteoarthritis of the cervical and dorsal spine, nine had diabetes mellitus, two had rheumatoid arthritis, one had systemic erythematous lupus, and seven had thyroid disease.

Demographic data, body mass index $\left(\mathrm{kg} / \mathrm{m}^{2}\right)$, and breast size were assessed. Breast size was determined by the distance, in centimeters, between each nipple to sternal notch, with the patient sitting and unclothed; the average of both breasts was used in the data analysis.

CTS evaluation was performed before surgery. CTS was defined by co-existence of symptoms, two physical examination findings (positives Tinnel sign and Phalen Test) and electrophysiological evaluation. Positive symptoms were defined as numbness, tingling, pain and weakness affecting the radial palm and the three radial fingers and the radial side of the fourth. Reduced manual dexterity with frequent object dropping was considered another positive symptom. ${ }^{11,15-17}$
Tinnel sign was considered positive when paresthesia in the distribution of the median nerve was elicited by tapering on the distal wrist crease over the median nerve. Phalen test was positive when the same symptoms described above where obtained after flexing the wrist $90^{\circ}$ for 60 seconds.

All women with a positive clinical evaluation were asked to undergo electrophysiological evaluation, once the main objective of the neurophysiological assessment of a patient with supposed CTS is to confirm the clinical suspicion suggested by history and clinical examination.

One year after reduction mammaplasty has been performed, the 22 patients that had been previously diagnosed CTS were called to a re-evaluation. Two of the patients were unable to participate in assessment and five patients had undergone carpal tunnel release surgery in a different hospital (five to nine months after mammaplasty surgery). CTS evaluation of the remaining 15 patients was based on the presence of CTS symptoms and positive Tinnel and Phalen tests. The other 75 patients who did not have CTS were also evaluated, one year after breast reduction, for occurrence of CTS signs and symptoms.

Statistical analysis was performed with the Statistical Package for the Social Sciences (SPSS). The Mann-Whitney test was used to compare both the normal condition and CTS patients, and to determine whether the distribution of individual variables measured differed. Pearson's coefficient correlation was used to ascertain the relationship between the prevalence of CTS and the variables: age, BMI and nipple-tosternal notch line.

\section{RESULTS}

Demographic characteristics of the patients are listed in Table 1. The mean age of the group was 38.8 years (range, 21-62 years), the majority $(92 \%)$ of the women were Caucasian $(n=89$ patients) and $8 \%$ were African ( $\mathrm{n}=8$ patients).

Body mass index (BMI) of the study group ranged from 19 to $37 \mathrm{~kg} / \mathrm{m}^{2}$, with a mean value of $28.5 \mathrm{~kg} / \mathrm{m}^{2}$. The mean value

Table 1 Demographic Data.

\begin{tabular}{|c|c|c|c|c|c|c|c|}
\hline \multirow[b]{2}{*}{ Variable } & \multicolumn{2}{|c|}{ Patients $(n=97)$} & \multicolumn{2}{|c|}{ Without CTS $(n=75)$} & \multicolumn{2}{|c|}{ With STC $(n=22)$} & \multirow[b]{2}{*}{$p$ Value* } \\
\hline & Mean & Range & Mean & Range & Mean & Range & \\
\hline Age (years) & 38.8 & $21-62$ & 37.1 & $21-61$ & 41.7 & $62-25$ & 0.001 \\
\hline BMI $\left(\mathrm{kg} / \mathrm{m}^{2}\right)$ & 28.6 & $19-37$ & 28.1 & $19-37$ & 30.2 & $22-37$ & 0.108 \\
\hline Breast size $(\mathrm{cm})$ & 35.9 & $25.2-52$ & 34.0 & $25.2-51.6$ & 42.3 & $31.3-52.3$ & 0.001 \\
\hline
\end{tabular}

*Mann-Whitney test comparing the groups with or without CTS. 
for breast size was $35.9 \mathrm{~cm}$ with a maximum value of $52.3 \mathrm{~cm}$ and a minimum of $25.2 \mathrm{~cm}$. The parameters found in this study sample are similar to other characteristics reported in other series of women undergoing reduction mammaplasty. ${ }^{3}$

Of the 97 patients included in the study, 22 patients (22.7\%) reported symptoms and electrophysiological evidence of CTS. From this group of 22 patients, five patients had a negative Tinnel test and two of these five patients had a negative Phalen test concomitantly. From the remaining 75 patients without any neurological upper limb complains, positive Tinnel sign was found in four women. Phalen test was positive in seven women and both tests were negative in 64 patients (Table 2).

Table 1 lists the comparison between women with and without CTS concerning the main variables: age $(p=0.001)$ and nipple-to-sternal notch line $(p=0.001)$ were associated with prevalence of CTS. In contrast BMI showed no relationship with the prevalence of CTS $(p=0.108)$. Correspondingly, it was found a significant positive correlation (Pearson's coefficient) between age and CTS prevalence $(p=0.001)$ and between breast size and CTS prevalence $(p=0.001)$. On the contrary BMI was not significantly associated with CTS prevalence $(p=0.077)$.

From the 15 patients with CTS diagnosis reassessed one year after reduction mammaplasty: five patients (33.3\%) reported absence of CTS symptoms, two of them had positive Phalen test and one had a positive Tinnel test; four patients (26.7\%) referred persistence of all symptoms, three of them presented positive Tinnel and Phalen tests and one had only positive Phalen test; four patients (26.7\%) had improvement of CTS symptoms but all had positive Phalen and Tinnel tests and two patients (13.3\%) reported worsening of the CTS symptoms with both having positive findings in clinical tests (Table 3). There were no statistically significant differences concerning age, breast size or BMI among the different symptom groups.

Table 2 CTS Evaluation.

\begin{tabular}{lcc}
\hline & $\begin{array}{c}\text { Without CTS } \\
(\boldsymbol{n}=\mathbf{7 5})\end{array}$ & $\begin{array}{c}\text { With CTS } \\
(\boldsymbol{n}=\mathbf{2 2})\end{array}$ \\
\hline Carpal Tunnel Symptoms & 0 & $22(100 \%)$ \\
Positive Tinel Sign & $4(5.3 \%)$ & $17(77.3 \%)$ \\
Positive Phalen Test & $7(9.3 \%)$ & $20(90.9 \%)$ \\
Negative Tinel and Phalen Tests & $64(85.3 \%)$ & $2(9.1 \%)$ \\
Positive Electrophysiology Studies & $2(2.6 \%)$ & $22(100 \%)$ \\
\hline
\end{tabular}

Table 3. CTS Evaluation One Year After Mammaplasty Reduction Surgery ( $n=15$ Patients).

\begin{tabular}{lcccc}
\hline & \multicolumn{4}{c}{ CTS Symptoms } \\
\cline { 2 - 5 } Variable & $\begin{array}{c}\text { Asymptomatic } \\
(\boldsymbol{n}=\mathbf{5})\end{array}$ & $\begin{array}{c}\text { Improved } \\
(\boldsymbol{n}=\mathbf{4})\end{array}$ & $\begin{array}{c}\text { Similar } \\
(\boldsymbol{n}=\mathbf{4})\end{array}$ & $\begin{array}{c}\text { Worsened } \\
(\boldsymbol{n}=\mathbf{2})\end{array}$ \\
\hline Age (years) & 38.6 & 42.25 & 43.75 & 53.5 \\
BMI $\left(\mathrm{kg} / \mathrm{m}^{2}\right)$ & 27.4 & 28.25 & 35.5 & 22.5 \\
Breast size $(\mathrm{cm})$ & 39.08 & 46.15 & 40.02 & 47.2 \\
\hline
\end{tabular}

From the initial group of 22 patients diagnosed CTS, the five patients who had undergone carpal tunnel release surgery in a different hospital were unable to participate in the assessment performed by the authors one year after breast surgery, thus could not be included in the final analysis.

\section{DISCUSSION}

Several conditions are known to be associated with CTS, like diabetes mellitus, rheumatoid arthritis, hypothyroidism, Colles fracture and pregnancy among others and the major part of the CTS causes remain unknown. The possibility of an association between macromastia and CTS has been studied in the past. According to some authors the prevalence of this neurological syndrome appears to be greater in women with larger breast: Pernia et al. demonstrate a 19.9\% incidence of CTS in a cohort of women with macromastia ${ }^{3}$ and Iwuagwu et al. reported a positive association with a $22 \%$ incidence of CTS among women with macromastia. ${ }^{4}$ Our study reinforces the data already published in the literature, we found a positive association with a $22.7 \%$ incidence of CTS among women with macromastia which is a significant by higher value as compared with the known population prevalence of $3 \%$ from epidemiologic studies. ${ }^{4,11}$

The most accurate diagnostic information of CTS is the combination of symptoms, positive findings in clinical examination and electrophysiologic data. ${ }^{4,11}$ Diagnosis based exclusively on electromyography test is discouraged. ${ }^{15}$ The incidence of false negatives, in fact, is statistically significant and data reported in literature ranges between $8 \%$ and $12 \%$. 0 n the other hand, the instrumental may register subclinical conduction alterations which do not require surgical treatment (false positives). ${ }^{16,17}$ Alternatively, the combination of Phalen and Tinel tests alone has limited ability to predict the electrodiagnosis. ${ }^{8}$ Following the same methodology of Iwuagwu 
et al. in their work we performed a CTS evaluation based in the three items described above.

In what concerns evaluated demographic data, race by itself is not known to be a risk factor for CTS; however, in our study it was impossible to evaluate its influence since the Caucasian women represent $92 \%$ of the study population. Nevertheless, our findings indicate an increased prevalence of CTS among women with macromastia. Statistical tests (Mann-Whitney) comparing the normal condition group of women and the CTS group confirmed a positive and significant association between breast size and prevalence of CTS as well as between age and CTS. Therefore this study supports recent published data and provides additional information relating to this particular association of variables: breast size and CTS prevalence. In what concerns BMI, our work confirms and supports the previously reported observations of Iwuagwu et al. in their study, where the association between BMI and CTS prevalence was not confirmed.

Concerning the 15 patients reassessed one year after mammaplasty reduction surgery, our findings suggests that it might be possible to achieve a partial or complete resolution of the neurological condition of the hand, in particular of the CTS, with a breast reduction procedure in women with macromastia. Five patients representing $33.3 \%$ of the sample reported complete resolution of the symptoms. Although clinical tests were positive in three of the patients, both Tinnel and Phalen test were not concomitantly positive in the same patient. Other four patients $(26.7 \%)$ had an improvement of CTS symptoms, so we can say that $60 \%$ of our 15 -patient sample have beneficiated with the surgical procedure in what concerns CTS.

The explanation why large breast should lead to the development of upper limb neurological symptoms is still unclear, although it is possible that the weight of the breasts in macromastia patients predisposes to some degree of upper limb venous congestion and, consequently edema formation. Kaye, although describing symptoms in an ulnar nerve distribution, attributed the findings in his patient to a type of thoracic outlet syndrome. ${ }^{8}$ The distraction forces imposed by the hypertrophied breasts could move (through the action of pectoralis minor muscle) the coracoid forward and by doing so, the breast could narrow the space through which the braquial plexus travels.

The fact that $60 \%$ of the 15 patients with CTS diagnosis experienced an improvement (26.7\%) or a complete resolution (33.3\%) of the neurological hand symptoms after mammaplasty reduction surgery emphasizes the existence of some mechanism responsible for the association of both pathologies.

On the other hand, six patients corresponding to $40 \%$ of the sample had referred persistence (four patients) or aggravation (two patients) of the neurological complains. These facts might have different interpretations: breast volume had no previous contribution to the neurological condition which remained stable in four of the patients and have evolved in two other patients; there was a pathological contribution of the breast dimension to the CTS development and irreversible alterations had occurred precluding the improvement of the symptoms following breast surgery; the breast condition had contributed to upper limb congestion and inflammatory alterations that could no longer be reverted with surgery and thus perpetuate the pathologic environment for CTS development and aggravation. A more proximal cause (e.g. cervical spondylosis) could not be excluded with certainty in these patients once the electrophysiological evaluation was not repeated one year after surgery.

Although this work followed the same methodological principles and diagnostic tests previously employed by other authors, ${ }^{4}$ it reinforces the data previously reported by using a larger and more representative sample of women with macromastia. The follow-up evaluation (one year after the reduction mammaplasty) was conducted in a small size sample which appears to be the main limitation of this study. These preliminary results, obtained from a 15-patient group, strengthens the probability of a causal association between both conditions and reveals the need for further investigation in to this particular area.

\section{CONCLUSION}

Women with macromastia appear to have an increased risk of having carpal tunnel syndrome, greater than reported from previous epidemiologic studies of prevalence in society. Breast size emerges as a positive risk factor to the development of CTS and a more accurate significant predictor of carpal tunnel syndrome when compared to body mass index. A positive causal association between breast size and carpal tunnel syndrome appears to exist according to the follow-up preliminary data.

\section{References}

1. Boschert MT, Barone CM, Puckett CL, Outcome analysis of reduction mammaplasty, Plast Reconst Surg 98:451-454, 1996. 
2. Dancey A, Khan M, Dawson J et al., Gigantomastia: A classification and review of the literature, J Plast Reconstr Aesthet Surg 61:493-502, 2008.

3. Pernia LR, Ronel DN, Leeper JD et al., Carpal tunnel syndrome in women undergoing reduction mammaplasty, Plast Reconst Surg 105:1314-1319, 2000.

4. Iwuagwu 0, Bajalan A, Platt A et al., Macromastia and carpal tunnel syndrome: Is there an association?, Aesth Plast Surg 30:535-537, 2006.

5. Netscher DT, Meade RA, Goodman CM et al., Physical and psychological symptoms among 88 volunteer subjects compared with patients seeking plastic surgery procedures to the breast, Plast Reconst Surg 105:2366-2373, 2000

6. Kerrigan CL, Collins ED, Kneeland TS et al., Measuring health state preferences in women with breast hypertrophy, Plast Reconst Surg 106:280-288, 2000.

7. Brinton LA, Persson I, Boice D et al., Breast cancer risk in relation to amount of tissue removed during breast reduction operations in Sweden, Cancer 91:478-483, 2001.

8. Kaye B, Neurological changes with excessive large breasts, South Med J 65:177-180, 1972.

9. Brown DM, Young VL, Reduction mammaplasty for Macromastia, Aesth Plast Surg 17:211-223, 1993.
10. Schnur P, Schnur D, Petty P et al., Reduction mammaplasty: An outcome study, Plast Reconstr Surg 100:875-883, 1997.

11. D'Arcy CA, McGee S, The rational clinical examination: Does this patient have carpal tunnel syndrome?, JAMA 283:3110-3117, 2000; erratum, JAMA 284:1384, 2000.

12. Ohnari K, Uozumi T, Tsuji S, Occupation and carpal tunnel syndrome, Brain Nerve 59:1247-1252, 2007.

13. Atroshi I, Gummesson C, Johnsson R et al., Prevalence of carpal tunnel syndrome in a general population, JAMA 282:153-158, 1999.

14. Glatt SB, Sarwer DB, O'Hara DE, Harmori C, Bucky LP, LaRossa D, A retrospective study of changes in physical symptoms and body image after reduction mammaplasty, Plast Reconstr Surg 103:76-82, 1999.

15. Louis D, Hankin F, Symptomatic relief following carpal tunnel decompression with normal electroneuromyographic studies, Orthopedics 10:434-436, 1987.

16. Phalen G, Gardner W, LaLonde A, Neuropathy of the median nerve due to compression beneath the trasverse carpal ligament, J Bone Joint Surg 32:109-112, 1950.

17. Kanz JN, Larson M, Fossel A et al., Validation of surveillance case definition of carpal tunnel syndrome, Am J Public Health 81:189, 1991. 
Copyright of Hand Surgery is the property of World Scientific Publishing Company and its content may not be copied or emailed to multiple sites or posted to a listserv without the copyright holder's express written permission. However, users may print, download, or email articles for individual use. 